\title{
Neutrophil-derived microRNAs put the (DNA) breaks on intestinal mucosal healing
}

\author{
Eóin N. McNamee \\ Mucosal Inflammation Program, School of Medicine, University of Colorado - Anschutz Medical Campus, Aurora, Colorado, USA. Institute of Immunology, Department of Biology, Maynooth University, \\ County Kildare, Ireland.
}

\begin{abstract}
A predominant feature of intestinal inflammation is the accumulation of neutrophils, which dictates a fine balance between epithelial repair or progression to chronic inflammation. While the processes of mucosal healing are well studied, how neutrophils advance an inflammatory insult towards epithelial neoplasia is less understood. In this issue of the $J \mathrm{Cl}$, Butin-Israeli et al. outline a mechanism whereby neutrophils control epithelial fitness and genomic instability via delivery of miR-23a-and miR-155-containing microparticles. Localized delivery of antisense oligonucleotides targeting miR-23a and miR-155 reversed this genomic instability and accelerated mucosal healing. This mechanism of neutrophilderived microRNA shuttling opens up new therapeutic potential to enhance epithelial healing and limit mucosal injury.
\end{abstract}

much lower cumulative CRC risks for UC patients over a 30-year period (9), though subgroups of patients, including those with early onset diagnosis, longer disease duration, or secondary complications such as primary sclerosing cholangitis (PSC), were at increased risk of CRC. As such, understanding how episodes of active inflammation in the colonic mucosa impact epithelial health and genomic integrity remains critically important. A consensus understanding has been that chronic episodes of localized neutrophilic inflammation is a contributing factor, resulting in oxidative stress-induced DNA damage, oncogene activation, and tumor progression $(10,11)$. However, an exact mechanistic understanding of the processes driving CRC in UC patients remains to be determined.

In this issue, Butin-Israeli and colleagues advance this knowledge gap by identifying a potentially novel and unexpected mechanism, whereby transmigrating neutrophils promote the accumulation of double-strand DNA breaks (DSBs) in intestinal epithelial cells by shuttling microRNA-containing microparticles (12). Patients with active inflammatory bowel disease (IBD) exhibited a marked accumulation of DSBs in intestinal epithelial cells, with a concomitant decrease in nuclear integrity. Moreover, these alterations were accompanied by increased expression of MPO, elastase, and inflammatory cytokines, such as TNF, IL-6, and IFN- $\gamma$. Using a series of reductionist in vitro culture systems, including human colonic enteroids and in vivo models of biopsy-induced sterile injury, Butin-Israeli et al. demonstrate that neutrophil-derived microparticles can selectively induce downstream effects on DNA damage. Neutrophil-derived microparticles alone induced DNA damage response in proliferating epithelial cells, a response that was blocked following inhibition of microparticle uptake. A decrease in epithelial cell proliferation did not 
A

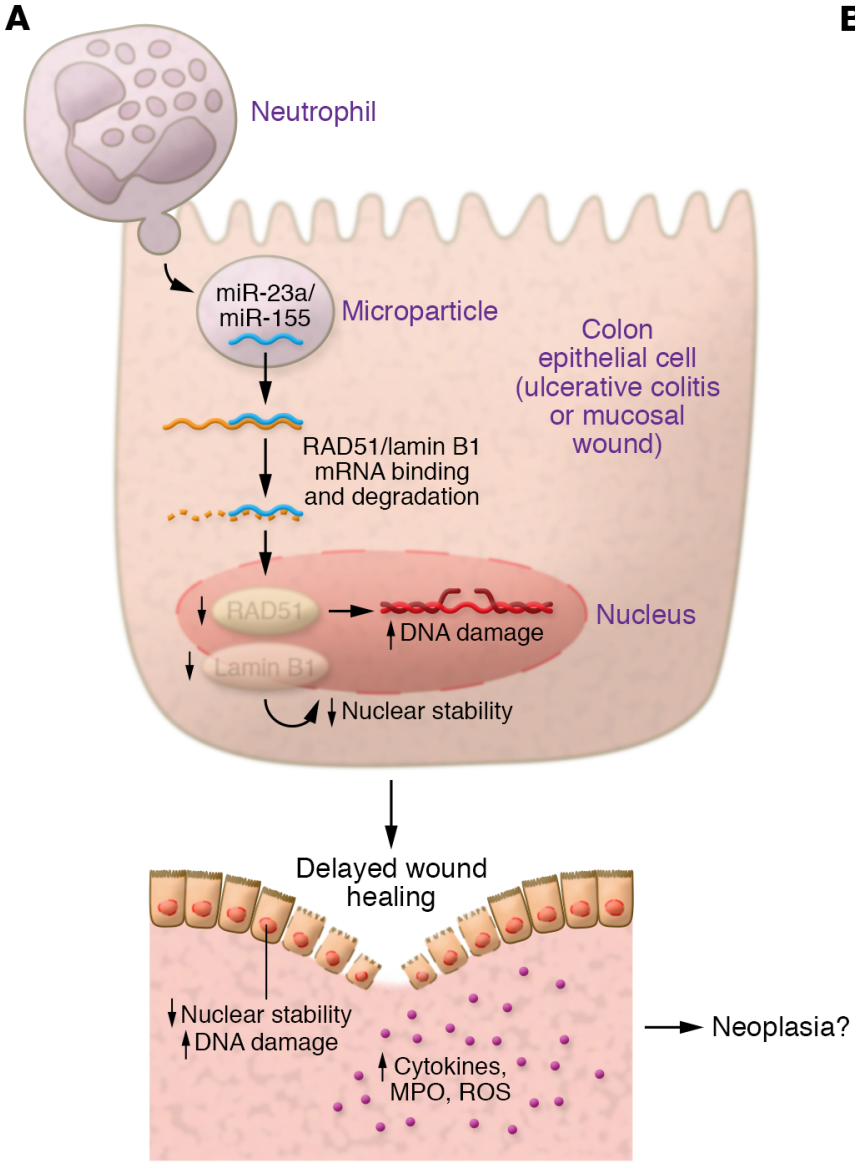

B

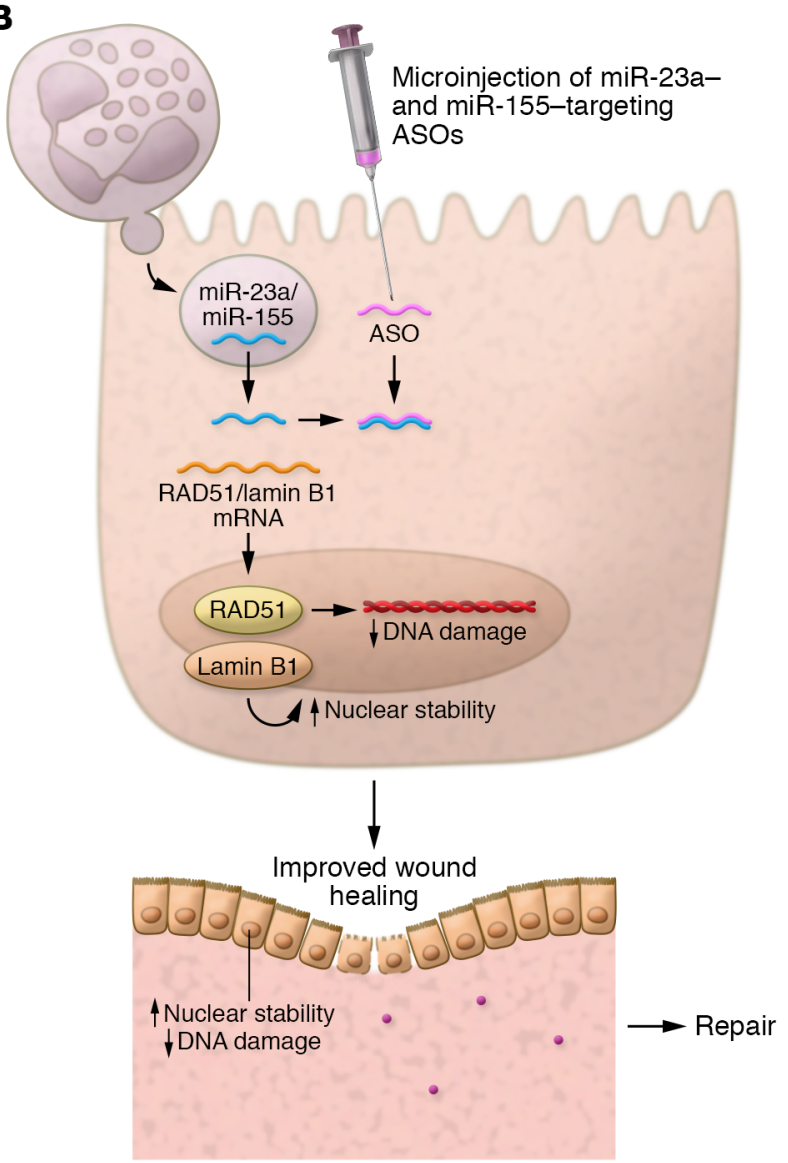

Figure 1. Therapeutic blockade of neutrophil-derived microRNAs limits epithelial DNA double-strand breaks and enhances intestinal mucosal healing. Neutrophil recruitment is a cardinal sign of intestinal inflammation, such as is seen with ulcerative colitis or mucosal wounding, and is associated with increased cytokine production, MPO, and ROS production. (A) At the injury site, neutrophils deliver microparticles containing miR-123a and miR-155 to intestinal epithelial cells. These microRNAs promote degradation of the mRNAs encoding the nuclear envelop protein lamin B1 and the DNA mismatch repair protein RAD51, leading to an increase in epithelial genomic instability, delayed wound healing, and possible neoplastic transformation. (B) Microinjection of antisense oligonucleotides (ASOs) targeting miR-123a and miR-155 prevent these miRNAs from binding their targets, thereby restoring expression of RAD51 and lamin B1 mRNA, improving genomic stability and promoting wound repair.

appear to be accompanied by induction of senescence or apoptosis, but it was associated with shortened newly synthesized DNA fibers, suggesting that neutrophil-derived microparticles regulated the integrity of DNA elongation. The authors further identified two microRNAs (miR-23a and miR-155) that are shuttled within these microparticles and directly inhibit production of RAD51, a critical DNA-repair protein, and nuclear envelope protein lamin B1. RAD51 catalyzes DNA strand exchange between homologous DNA partners, and is therefore essential for repairing DNA breaks by guiding repair templates. Importantly, pretreatment of epithelial cells with antisense oligonucleotides (ASOs) against miR-23a and miR-155 prevented neutrophil microparticle-associated DNA damage, demonstrating a selective effect.
MicroRNAs are small noncoding RNAs ( 22 nucleotides) that regulate an array of cellular processes by inhibiting the translation of protein-coding mRNA transcripts. Functional microRNAs encapsulated within microparticles and microvesicles have been shown to shuttle into target cells in mucosal tissues during injury, inflammation, and infection (13-15); however, selectively targeting specific microRNAs in vivo to a defined cell type is technically very challenging (Figure 1).

\section{Targeting candidate microRNA for mucosal healing: no small task}

In addition to in vitro systems and murine models, Butin-Israeli et al. demonstrated that expression of both miR-23a and miR155 is increased in mucosal biopsies from patients with active IBD and correlate with increased neutrophils, defective epithelial micronuclei, increased epithelial DNA damage, and decreased lamin B1. One of the greatest practical challenges in developing a proof-of-concept therapeutic modality to target these microRNAs is selective targeting to epithelial cells in the intestine. The authors overcame this hurdle by utilizing an elegant model of inflammation-independent wounding (needle biopsy with mouse colonoscopy) and delivered ASOs targeting miR-23a and miR-155 by site-directed microinjection 24 hours after injury (12). This approach substantially protected the wound site from DSBs and markedly increased expression of lamin B1 and RAD51, and enhanced epithelial restitution and the rate of wound closure (Figure 1). It is worth noting that 
off-target effects of microRNA blockade could have profound long-term consequences for epithelial function. For example, miR-155 is known to repress hypoxia-inducible factor HIF-1 $\alpha$, which has a protective role in maintaining intestinal epithelial barrier integrity (16-18). Thus, blocking miR-23a and miR-155 with ASOs may impact other reparative gene expression profiles. In addition, it is not known how this approach works in the context of inflammation and microbiome dysbiosis, such as occurs during active flares in UC patients, and it may yield different results. However, it should be noted that antibody-mediated depletion of neutrophils had similar effects as ASO-mediated blockade of miR-23a and miR-155. Acute depletion of neutrophils in a dextran sulphate sodium-induced model of colitis reduced DSBs and reversed the imbalance of lamin B1 and RAD51. Thus, ButinIsraeli and colleagues have identified a remarkably powerful miR-23a/miR-155/ lamin B1/RAD51 axis as a reparative pathway that warrants further investigation.

\section{Microparticle-derived microRNAs: the how, why, and what's next}

The linear focus of the Butin-Israeli et al. study on neutrophil-derived microparticles makes clinical sense, considering that accumulation of transepithelial neutrophils within colonic crypts is the predominant pathological hallmark of active UC (2). However, during UC, there is a multitude of innate leukocytes, including monocytes, macrophages, and eosinophils, present that are also capable of secreting microparticles and expressing distinct microRNAs. An intriguing question raised by this work is why are specific microRNAs selectively packaged into microparticles by discrete stimuli (i.e., only expressed in activated neutrophils)? This question is further emphasized in the work of Butin-Israeli and colleagues, as another RAD51-targeting miRNA, miR103, which is also increased in activated neutrophils, was not incorporated into neutrophil-derived microparticles. In contrast, recently published data demonstrate that neutrophil-derived microparticles containing miR-223 regulate lung epithelial cells in the setting of both sterile and infectious insults (14), whereas the injured lung epithelium regulates the recruitment of macrophage via microvesicle-derived miR-17 and miR-221 (13). The mechanistic understanding of these discrepant yet selective biological processes is sure to have major implications for our understanding of a multitude of inflammatory conditions.

The work of Butin-Israeli and colleagues also raises the question of whether all neutrophils (and neutrophil-derived microparticles) are created equally? That is to say, will multiple rounds of neutrophil hematopoiesis and consistent mucosal ulceration during active UC flares alter the fitness and function of microparticles? The stratification of neutrophils based on phenotype and function as pro- versus anticancer has been observed, and CRC can be stratified based on the composition of neutrophil and myeloid-derived suppressor cell (MDSC) infiltrates (19, 20). These phenotypic data are lacking, but warranted, for the continuum of chronic mucosal disease in UC; however, immune cell phenotypes are sure to be confounded by continuing responses to the commensal microbiota and UC-associated dysbiosis.

Mucosal healing and remission are emerging as the primary therapeutic goals for preventing CRC in UC patients $(21,22)$. The work by Butin-Israeli et al. raises the question of how treatment responses of UC patients to an armamentarium of current therapeutics might alter the identified neutrophil/microparticle/epithelial cell axis? Recent analysis has suggested that current therapies, such as 5-aminosalicylic acid (5-ASA) and thiopurine, have chemopreventive effects in UC $(23,24)$, but there is limited evidence on the effects of biologic therapy on the incidence of CRC for UC patients. To date, it is not known how these biotherapeutics impact microparticle and microRNA biology. As eluded to by Butin-Israeli and colleagues, the utility of future microRNA therapeutics will always be hampered by the technical challenges of cell-specific targeting and subverting the issue of multiple off-target mRNA candidates for each miRNA. However, by utilizing selective microRNAs as a prism to separate and underscore major disease pathways in vivo, elegant bodies of work such as the one by Butin-Israeli and colleagues can uncover master circuits driving disease in both IBD and CRC. While both microRNA and microparticle biology are relatively understudied, extensions of Butin-Israeli et al.'s work may identify drug-responsive therapeutic candidates for both IBD and CRC.

\section{Acknowledgments}

ENM is supported by grants from the Crohn's and Colitis Foundation of America (409992) and the NIH National Institute of Diabetes and Digestive and Kidney Diseases (R01DK111856).

Address correspondence to: Eóin N. McNamee, Institute of Immunology, Department of Biology, Maynooth University, County Kildare, Ireland. Phone: 353.1.708.6369; Email: eoin.n.mcnamee@mu.ie.

1. Wéra O, Lancellotti P, Oury C. The dual role of neutrophils in inflammatory bowel diseases. J Clin Med. 2016;5(12):E118.

2. Campbell EL, et al. Transmigrating neutrophils shape the mucosal microenvironment through localized oxygen depletion to influence resolution of inflammation. Immunity. 2014;40(1):66-77.

3. Flannigan KL, et al. IL-17A-mediated neutrophil recruitment limits expansion of segmented filamentous bacteria. Mucosal Immunol. 2017;10(3):673-684.

4. Kühl AA, et al. Aggravation of different types of experimental colitis by depletion or adhesion blockade of neutrophils. Gastroenterology. 2007;133(6):1882-1892.

5. Brazil JC, Louis NA, Parkos CA. The role of polymorphonuclear leukocyte trafficking in the perpetuation of inflammation during inflammatory bowel disease. Inflamm Bowel Dis. 2013;19(7):1556-1565.

6. Chin AC, Parkos CA. Neutrophil transepithelial migration and epithelial barrier function in IBD: potential targets for inhibiting neutrophil trafficking. Ann N Y Acad Sci. 2006;1072:276-287.

7. Lopez A, Pouillon L, Beaugerie L, Danese S, Peyrin-Biroulet L. Colorectal cancer prevention in patients with ulcerative colitis. Best Pract Res Clin Gastroenterol. 2018;32-33:103-109.

8. Jess T, Rungoe C, Peyrin-Biroulet L. Risk of colorectal cancer in patients with ulcerative colitis: a meta-analysis of population-based cohort studies. Clin Gastroenterol Hepatol. 2012;10(6):639-645.

9. Jess T, Simonsen J, Jørgensen KT, Pedersen BV, Nielsen NM, Frisch M. Decreasing risk of colorectal cancer in patients with inflammatory bowel disease over 30 years. Gastroenterology. 2012;143(2):375-381.e1; quiz e13.

10. Risques RA, et al. Ulcerative colitis-associated colorectal cancer arises in a field of short telomeres, senescence, and inflammation. Cancer Res. 2011;71(5):1669-1679.

11. Rutter M, et al. Severity of inflammation is a risk factor for colorectal neoplasia in ulcerative colitis. Gastroenterology. 2004;126(2):451-459.

12. Butin-Israeli V, et al. Neutrophil-induced genomic instability impedes resolution of inflammation and wound healing. JClin Invest. 
2019;129(2):712-726.

13. Lee H, Zhang D, Wu J, Otterbein LE, Jin Y. Lung epithelial cell-derived microvesicles regulate macrophage migration via microRNA-17/221induced integrin $\beta 1$ recycling. J Immunol. 2017;199(4):1453-1464.

14. Neudecker V, et al. Neutrophil transfer of miR-223 to lung epithelial cells dampens acute lung injury in mice. Sci Transl Med. 2017;9(408):eaah5360.

15. Zhang J, et al. Exosome and exosomal microRNA: trafficking, sorting, and function. Genomics Proteomics Bioinformatics. 2015;13(1):17-24.

16. Bruning U, et al. MicroRNA-155 promotes resolution of hypoxia-inducible factor 1alpha activity during prolonged hypoxia. Mol Cell Biol. 2011;31(19):4087-4096

17. Colgan SP, Taylor CT. Hypoxia: an alarm signal during intestinal inflammation. Nat Rev Gastroenterol Hepatol. 2010;7(5):281-287.

18. Karhausen J, Furuta GT, Tomaszewski JE, Johnson RS, Colgan SP, Haase VH. Epithelial hypoxia-inducible factor- 1 is protective in murine experimental colitis. J Clin Invest. 2004;114(8):1098-1106.

19. Fridlender ZG, et al. Polarization of tumor-associated neutrophil phenotype by TGF- $\beta$ : "N1" versus "N2" TAN. Cancer Cell. 2009;16(3):183-194.

20. Elliott LA, Doherty GA, Sheahan K, Ryan EJ. Human tumor-infiltrating myeloid cells: phenotypic and functional diversity. Front Immunol. 2017;8:86.

21. Bryant RV, et al. Beyond endoscopic mucosal healing in UC: histological remission better predicts corticosteroid use and hospitalisation over
6 years of follow-up. Gut. 2016;65(3):408-414. 22. Shah SC, Colombel JF, Sands BE, Narula N. Mucosal healing is associated with improved long-term outcomes of patients with ulcerative colitis: a systematic review and meta-analysis. Clin Gastroenterol Hepatol. 2016;14(9):1245-1255.e8.

23. van Schaik FD, van Oijen MG, Smeets HM, van der Heijden GJ, Siersema PD, Oldenburg B. Thiopurines prevent advanced colorectal neoplasia in patients with inflammatory bowel disease. Gut. 2012;61(2):235-240.

24. Velayos FS, Terdiman JP, Walsh JM. Effect of 5 -aminosalicylate use on colorectal cancer and dysplasia risk: a systematic review and metaanalysis of observational studies. Am J Gastroenterol. 2005;100(6):1345-1353. 